\title{
Interdependence and contagion in five ASEAN countries and five developed countries in the area of financial linkages
}

\author{
N. Trihadmini \\ Universitas Katolik Indonesia Atma JayaJakarta, DKI Jakarta, Indonesia \\ T.A. Falianty \\ Department of Economics, Faculty of Economics and Business, Universitas Indonesia, Depok, Indonesia
}

\begin{abstract}
Financial globalisation brings great benefits but also results in the vulnerability of the economy. This study aims to analyse whether there were contagion effects of the 2008 global financial crisis or mere interdependence between five countries of the Association of Southeast Asian Nations (ASEAN) and five developed countries through financial channels. The analysis used mixed-frequency data and included foreign exchange markets, stock markets, policy interest rates, and money markets in the period 1990-2016. Crossmarket correlation, Dynamic Conditional Correlation (DCC), and vector auto regression (VAR) were also used in the analysis.

The DCC method indicated that there was no extreme increase in the DCC coefficient in the period after the crisis at variables of (1) exchange rates, (2) stock indices, and (3) money market rates; thus, the phenomenon of the relationship was interdependence and not contagion. However, the policy interest rate variable showed a significant increase in the DCC coefficient in the three periods of analysis (1990-1997, 1998-2007, 2008-2015). This indicates the presence of a strong relationship between the monetary policies of each country and those of the other countries and the increasing coordination of monetary policies among the five ASEAN countries in this study. Although the US monetary policy was used as a reference by the five ASEAN countries and the five developed countries still had a dominant influence on the ASEAN financial markets, the linkages through financial channels among the five ASEAN countries were relatively weak.
\end{abstract}

\section{INTRODUCTION}

Financial globalisation has brought enormous benefits to the economy, but these are comparable to the economic vulnerability caused. Countries worldwide are increasingly connected to each other through financial channels, trade, or other economic activities. Interdependence through financial channels results in capital and financial flows across borders. Liquidity constraints and loss on the financial markets result in recomposition and global turnaround assets. This asset turnover creates opportunities and threats, as a result of the close interconnection between countries.

In line with the increasingly integrated economy in the era of globalisation, financial crisis in one country can easily spread to other countries and become a global financial disaster within a short period of time. An economic crisis caused by financial turmoil leading to instability, especially when it happens continuously for a long time, will interfere with other sectors in the economy. When the financial system is large, the risk of volatility or crisis is also bigger; so, it can lead to vulnerability in the stability of a financial system.

Economic crises and their spread have resulted in huge economic costs, as already experienced by most countries worldwide, including five countries of the Association of Southeast Asian Nations (ASEAN; Indonesia, Malaysia, Thailand, Singapore, and Philippines). 


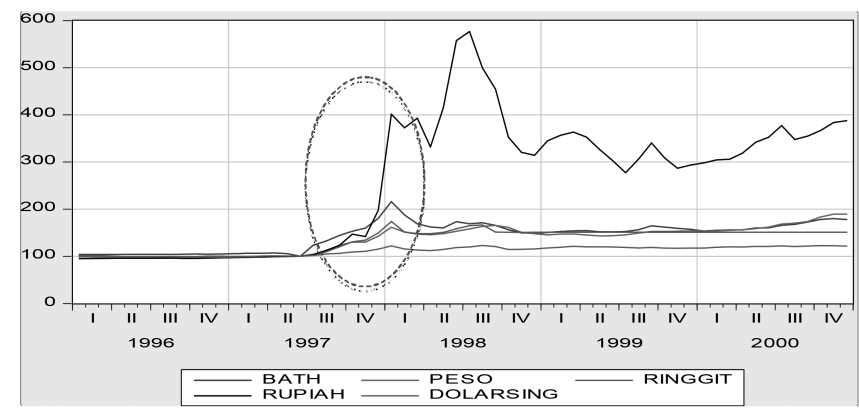

Figure 1. Exchange rate movement in five ASEAN countries during the 1997-98 financial crisis. Source: finance.yahoo.com (data processed).

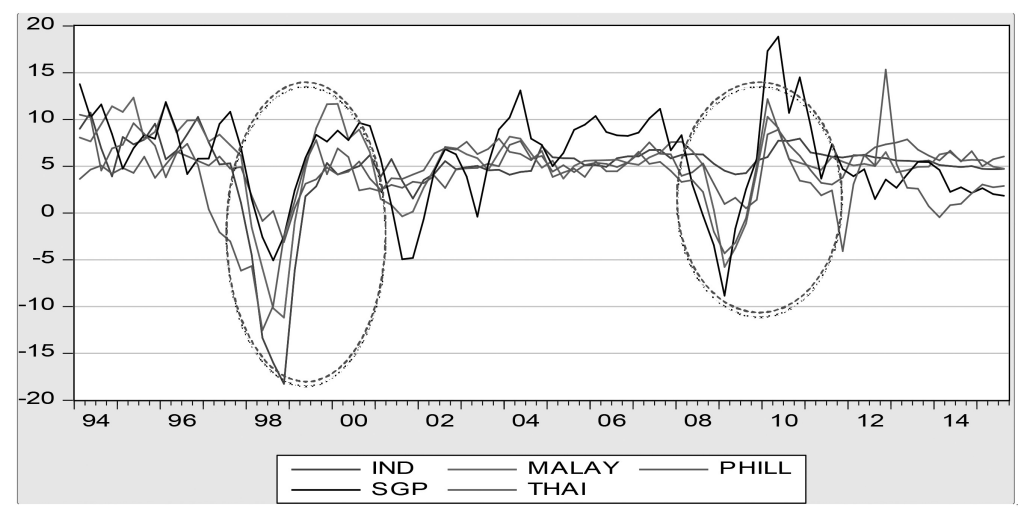

Figure 2. Economic growth in five ASEAN countries during the financial crisis in 1997 and 2008. Source: IFS, IMF (data processed).

Figure 1 shows that most of the five ASEAN countries experienced a sharp depreciation during the financial crisis of 1997-98. The depreciation had a widespread impact on the economy, which decreased purchasing power, aggregate demand, and ultimately output. In line with these conditions, Figure 2 shows the declining economic growth in five ASEAN countries as a result of the global financial crisis in 2008. The decrease in the national income lowered the quality of life in society as a whole. During these crises, interdependence and contagion of financial markets played a crucial role in the high level of integration between countries and financial markets. The interdependence became a major source of spillover effects, which led to extreme volatility and involved contagion effects.

Based on this background, this study aims to determine whether there were contagion effects of the 2008 global financial crisis or whether there was mere interdependence between five ASEAN countries and five developed countries through financial channels. This research is highly important because understanding the relationship or interconnection of financial channels allows analysis of the global mapping of risk, also known as concentrations of systemic risk, identification of transmission propagation of shocks, and improvement of the supervision of implementation in macro prudential policy.

\section{LITERATURE REVIEW}

\subsection{Interdependence and contagion}

There is a fundamental difference between interdependence and contagion. Referring to Forbes and Rigobon (2002), interdependence is co-movement during a stable period, driven 
by strong linkages between markets. It emphasises real linkages and fundamental integration as channels for transmission shocks between countries and markets in both crisis and noncrisis periods (Shen et al., 2015). Interconnection or financial linkage is a network of credit exposure, trade relations, or other linkages between economic activities and the interdependence of the financial agent (Gray, 2015). Finance interconnection is very important because it can serve as a channel of transmission (crisis). The impact of the failure of large interconnected entities can spread widely and rapidly throughout the financial system, thereby causing instability in the financial system worldwide. Financial interdependence can occur through the relationship between financial institutions, both banks and non-banks.

Meanwhile, Dornbusch et al. (2000) define contagion as a significant increase in crossmarket linkages after a shock to an individual country or a group of countries, measured by the degree to which asset prices and financial flows move together across markets relative to this co-movement in tranquil times. An increase in co-movement does not reflect irrational behaviour of investors. Forbes and Rigobon (2001) explain a more specific definition of contagion, which is a significant increase in cross-market correlation after a shock in one country or a group of countries. Cross-market linkages can be measured by the correlation of asset returns, the probability of a speculative attack to transmit shocks or volatility. Cleassens and Forbes (2004) state that when two countries are located in separate geographical areas, have different economic structures, and almost do not have a direct connection through the channels of trade for example, the propagation from one country to another is called contagion. Forbes and Rigobon (2002) propose the use of the definition of 'shift contagion', which is not merely a contagion to emphasise the occurrence of a significant change in cross-market linkages after a shock. Some economists argue that if a shock is transmitted from one country to another, even though there is no significant change in the cross-market linkage, this transmission is also called contagion, but it is not the shift-contagion.

Kaminsky et al. (2003) state that contagion is an episode where there is an instant or immediate significant effect in the aftershocks in several countries. This results in excess comovement in the economic and financial variables across countries as a response to the common shock in one country or a group of countries.

\subsection{Financial channel}

Several studies have been conducted to investigate contagion through financial channels. Gómez and Rivero (2014) found that the integration of bilateral debt and bond yields is an important channel that transmits a shock, although it is not proven that the integration of foreign direct investment will drive asset prices together. Stock market volatility is the variable most commonly found to spread out the crisis, as stated by Luchtenberg, K.F. \& Vu, Q.V. (2015), Morales, L. \& O'Callaghan, B.,A., (2014), Kenourgios (2014), and Podlich, N., $\&$ Wedow, M. (2014). Ambiguity between interdependence and contagion was also investigated by Morales, L. \& O'Callaghan, B.,A., (2014), and Ahlgren, N. \& Antell, J. (2010) who state that there is no contagion effect of the US stock market on the global financial markets, but volatility is derived from the transmission of economic linkages between countries. They also state that there are short-term relationships between developed countries (United States, Germany, Japan, United Kingdom) and emerging markets (Hong Kong, Korea, Mexico), but that is not contagion. The short-term relationship has important implications on portfolio diversification.

Unsal, D.F. \& Jain-Chandra, S. (2012) states that global financial shocks are transmitted to domestic economies through three channels separately, namely (1) financial channels, (2) channels of trade, and (3) investors' perception channels through substitution effects. Financial channels can occur through financial spill over and also connectedness asset markets such as herding behaviour, which only exists or occurs in a crisis condition, which is generally called 'pure contagion' (Kaminsky and Reinhart, 2000).

Dungey and Tambakis (2003) also state that financial link transmission can occur through the currency, equity, and bond markets, where the patterns of interaction and transmission in one country and between countries can be described as follows. 
1. Interaction between currency and equity markets in the country.
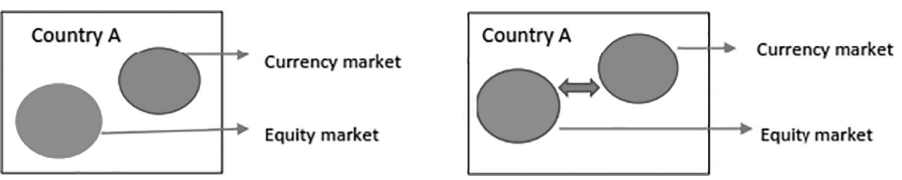

2. Several countries with similar patterns of interaction between currency andequity markets.
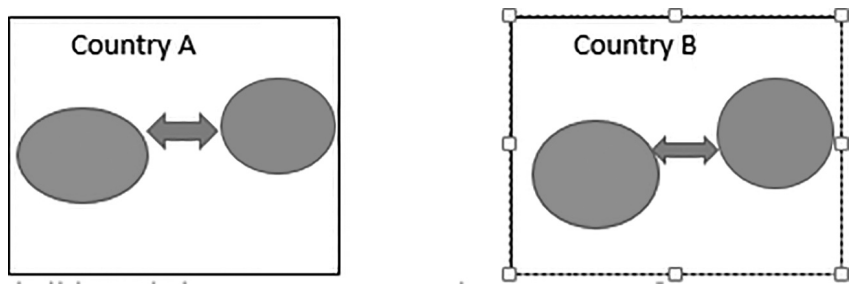

3. Interaction between country A and country B and their currency markets

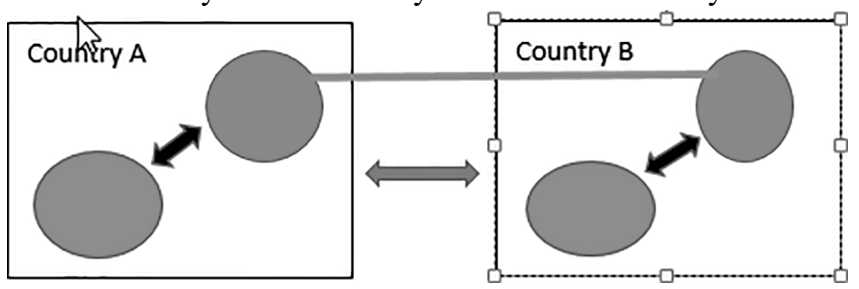

4. Interaction between the currency and equity markets of country A and country B.

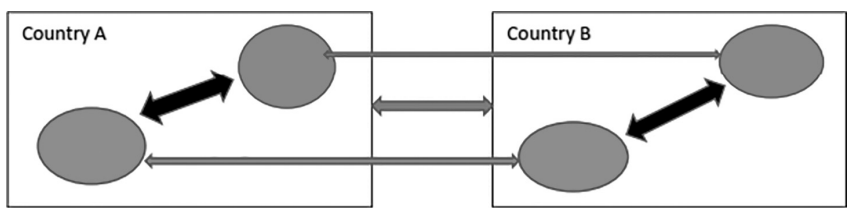

5. Interaction involving more than two countries.

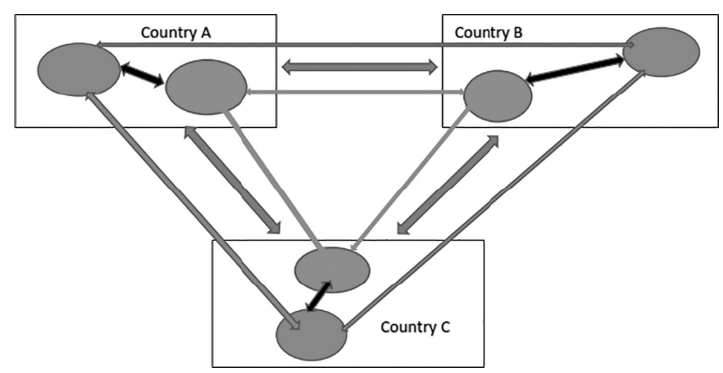

\section{METHODOLOGY}

The aim of this research is to analyse whether contagion effect occurs in times of crisis or whether there is only interdependence among five ASEAN countries (Indonesia, Malaysia, Thailand, Singapore, and Philippines) and five developed countries (United States, Europe Union, Japan, China, and Korea). The selection of the five ASEAN countries to represent the ASEAN profile is based on the consideration that the five countries have good economic growth compared with other ASEAN member states. In addition, they have high economic 
openness, so exposure to global shock could be propagated through them. The selection of the five developed countries is based on the consideration that these countries have great economic power and relative dominance, making them a potential source of shock in the global economy.

In analysing the dynamic relationships through financial linkages, financial variables such as exchange rates, stock indices, central bank policy rates, and money market rates were used. The selection of these research variables is based on the economic theory and the review of literature relating to contagion and propagation. For the purposes of the analysis, several analytical tools were used, namely (1) Dynamic Conditional Correlation (DCC), (2) Granger causality test, (3) Engle-Granger cointegration test, and (4) variance decomposition analysis. This study took different periods for the variables, between the years 1990 and 2016, and used monthly data published by the International Monetary Fund, World Bank, Asian Development Bank, and other relevant resources.

\section{EMPIRICAL RESULTS}

\subsection{Currency market}

Figure 3 illustrates the rolling standard deviation of the exchange rate movement in the five ASEAN countries and five developed countries. As seen on the Figure 3 graphs, there was similar movement, especially the one occurring in 2008, as a result of the global financial crisis. The South Korean Won, Indonesian rupiah, Philippine peso, Singaporean dollar, and Thai baht experienced the same fluctuation as the euro, Japanese yen, and Chinese yuan. All these currencies were already in US dollar units, so the movement of the US dollar is already included in the movement of the currency of each of these countries.

Exchange rate fluctuation in the same direction indicates the presence of global currency interconnection. There is still debate between contagion and interconnection. Forbes and Rigobon (2002) state that if the correlation coefficient between two or more countries is relatively high in the period of crisis and non-crisis, it indicates the occurrence of interdependence and not contagion.
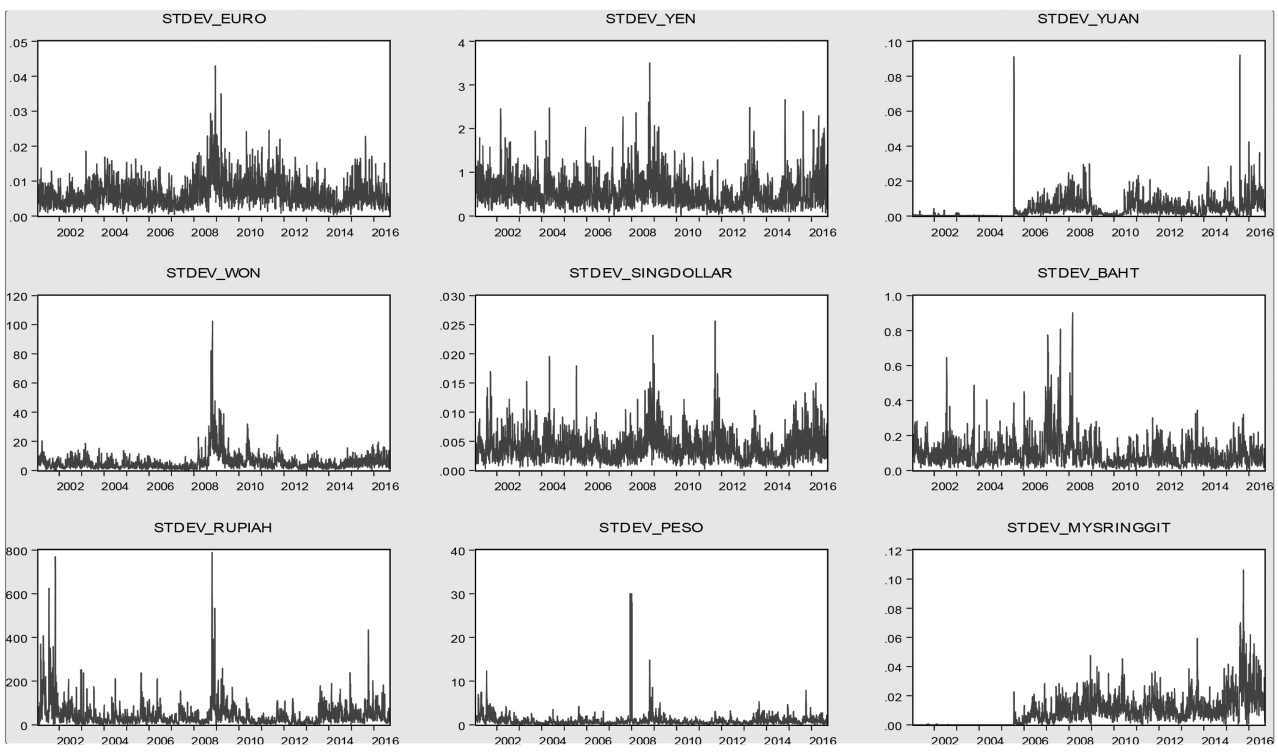

Figure 3. Rolling standard deviation of exchange rate movement (2000-16). Sources: IFS, World Bank (data processed). 
The statistical tests using DCC in Tables 1 and 2 show that for the ASEAN and developed countries, the relationship was interdependence and not contagion. The average coefficient of DCC was $12.86 \%$ prior to 2008 and $26.09 \%$ after the 2008 financial crisis. Although there was an increase in the coefficient of DCC in the period after the crisis, the increase did not occur significantly; thus, it cannot be stated as the occurrence of contagion.

Table 1. Dynamic Conditional Correlation (DCC) of exchange rates (January 2000-December 2007).

\begin{tabular}{|c|c|c|c|c|c|c|c|c|c|}
\hline & $\begin{array}{l}\text { STDEV_- } \\
\text { EURO }\end{array}$ & $\begin{array}{l}\text { STDEV_- } \\
\text { YEN }\end{array}$ & $\begin{array}{l}\text { STDEV_- } \\
\text { YUAN }\end{array}$ & $\begin{array}{l}\text { STDEV_- } \\
\text { WON }\end{array}$ & $\begin{array}{l}\text { STDEV }_{-} \\
\text {RUPIAH }\end{array}$ & $\begin{array}{l}\text { STDEV_- } \\
\text { PESO }\end{array}$ & $\begin{array}{l}\text { STDEV }_{-} \\
\text {SINGDO } \\
\text { LLAR }\end{array}$ & $\begin{array}{l}\text { STDEV_- } \\
\text { BAHT }\end{array}$ & $\begin{array}{l}\mathrm{AVG}_{-} \\
\text {GLOBAL }\end{array}$ \\
\hline $\begin{array}{l}\text { STDEV_- } \\
\text { EURO }\end{array}$ & 1.0000 & 0.2410 & -0.0537 & 0.1951 & -0.0100 & 0.0342 & 0.3373 & 0.0583 & \\
\hline $\begin{array}{l}\text { STDEV_- } \\
\text { YEN }\end{array}$ & 0.2410 & 1.0000 & 0.0714 & 0.3593 & 0.0618 & 0.0682 & 0.4124 & 0.2056 & \\
\hline $\begin{array}{l}\text { STDEV_- } \\
\text { YUAN }\end{array}$ & -0.0537 & 0.0714 & 1.0000 & -0.0834 & -0.0963 & 0.1492 & 0.0843 & 0.2632 & \\
\hline $\begin{array}{l}\text { STDEV_ } \\
\text { WON }\end{array}$ & 0.1951 & 0.3593 & -0.0834 & 1.0000 & 0.1069 & -0.0087 & 0.3934 & 0.1673 & \\
\hline $\begin{array}{l}\text { STDEV }_{-} \\
\text {RUPIAH }\end{array}$ & -0.0100 & 0.0618 & -0.0963 & 0.1069 & 1.0000 & 0.2320 & 0.1372 & -0.0212 & \\
\hline $\begin{array}{l}\text { STDEV }_{-} \\
\text {PESO }\end{array}$ & 0.0342 & 0.0682 & 0.1492 & -0.0087 & 0.2320 & 1.0000 & 0.0547 & 0.0336 & \\
\hline $\begin{array}{l}\text { STDEV }_{-} \\
\text {SINGDO } \\
\text { LLAR }\end{array}$ & 0.3373 & 0.4124 & 0.0843 & 0.3934 & 0.1372 & 0.0547 & 1.0000 & 0.2092 & \\
\hline $\begin{array}{l}\text { STDEV }_{-} \\
\text {BAHT }\end{array}$ & 0.0583 & 0.2056 & 0.2632 & 0.1673 & -0.0212 & 0.0336 & 0.2092 & 1.0000 & \\
\hline Sum & 0.8022 & 1.4198 & 0.3347 & 1.1300 & 0.4105 & 0.5633 & 1.6285 & 0.9159 & \\
\hline Average & 0.1146 & 0.2028 & 0.0478 & 0.1614 & 0.0586 & 0.0805 & 0.2326 & 0.1308 & 0.1286 \\
\hline
\end{tabular}

Note: STDEV, standard deviation; AVG, average.

Source: IFS, World Bank (data processed).

Table 2. DCC of exchange rates (January 2008-August 2016).

\begin{tabular}{|c|c|c|c|c|c|c|c|c|c|}
\hline & $\begin{array}{l}\text { STDEV_ } \\
\text { EURO }\end{array}$ & $\begin{array}{l}\text { STDEV_- } \\
\text { YEN }\end{array}$ & $\begin{array}{l}\text { STDEV } \\
\text { YUAN }\end{array}$ & $\begin{array}{l}\text { STDEV_ } \\
\text { WON }\end{array}$ & $\begin{array}{l}\text { STDEV }_{-} \\
\text {RUPIAH }\end{array}$ & $\begin{array}{l}\text { STDEV_- } \\
\text { PESO }\end{array}$ & $\begin{array}{l}\text { STDEV_ } \\
\text { SING } \\
\text { DOLLAR }\end{array}$ & $\begin{array}{l}\text { STDEV_- } \\
\text { BAHT }\end{array}$ & $\begin{array}{l}\mathrm{AVG}_{-} \\
\text {GLOBAL }\end{array}$ \\
\hline $\begin{array}{l}\text { STDEV_- } \\
\text { EURO }\end{array}$ & 1.000 & 0.312 & 0.073 & 0.418 & 0.217 & 0.194 & 0.553 & 0.205 & \\
\hline $\begin{array}{l}\text { STDEV_- } \\
\text { YEN }\end{array}$ & 0.312 & 1.000 & 0.063 & 0.386 & 0.244 & 0.258 & 0.279 & 0.154 & \\
\hline $\begin{array}{l}\text { STDEV_- } \\
\text { YUAN }\end{array}$ & 0.073 & 0.063 & 1.000 & 0.056 & 0.033 & 0.034 & 0.176 & 0.141 & \\
\hline $\begin{array}{l}\text { STDEV_- } \\
\text { WON }\end{array}$ & 0.418 & 0.386 & 0.056 & 1.000 & 0.398 & 0.355 & 0.527 & 0.145 & \\
\hline $\begin{array}{l}\text { STDEV }_{-} \\
\text {RUPIAH }\end{array}$ & 0.217 & 0.244 & 0.033 & 0.398 & 1.000 & 0.871 & 0.328 & 0.147 & \\
\hline $\begin{array}{l}\text { STDEV }_{-} \\
\text {PESO }\end{array}$ & 0.194 & 0.258 & 0.034 & 0.355 & 0.871 & 1.000 & 0.307 & 0.156 & \\
\hline $\begin{array}{l}\text { STDEV_ } \\
\text { SING } \\
\text { DOLLAR }\end{array}$ & 0.553 & 0.279 & 0.176 & 0.527 & 0.328 & 0.307 & 1.000 & 0.274 & \\
\hline $\begin{array}{l}\text { STDEV_- }_{-} \\
\text {BAHT }\end{array}$ & 0.205 & 0.154 & 0.141 & 0.145 & 0.147 & 0.156 & 0.274 & 1.000 & \\
\hline Sum & 1.972 & 1.696 & 0.576 & 2.286 & 2.238 & 2.174 & 2.445 & 1.223 & \\
\hline Average & 0.2818 & 0.2423 & 0.0823 & 0.3265 & 0.3197 & 0.3106 & 0.3492 & 0.1747 & 0.2609 \\
\hline
\end{tabular}

Source: IFS, World Bank (data processed). 
Table 3 indicates that bidirectional relationship between two currencies does not always occur. There was causality between the currencies of the five developed countries and those of the five ASEAN countries, except for the Malaysian ringgit. The Malaysian ringgit was relatively independent from the currencies of the developed countries, but there was causality with the regional currencies. Currency movements of the five ASEAN countries (except Malaysia) were relatively more influenced by the strong currencies of the developed countries compared with that of neighbouring countries in the region. Based on the Granger causality test, the exchange rate relationship can be mapped as shown in Table 3.

\subsection{Equity market}

Figure 4 shows that all the stock indices declined sharply during the global financial crisis, indicating a strong interconnection in the stock market. The Engle-Granger cointegration test showed that there was cointegration between all stock indices in the five ASEAN countries (except Thailand) and the five developed countries, both before and after the global financial crisis in 2008, as shown in Table 4.

Thus, Table 4 shows that cointegration or long-term relationship on the stock market occurred not only in the period after the crisis but also in the period before the crisis. This suggests that there is a naturally strong relationship to the stock market.

Table 3. Causality relationship of exchange rates (9 January 2001-25 August 2016).

\begin{tabular}{llllllllll}
\hline & & & & & SING & & & \\
& YUAN & YEN & WON & EURO & DOLLAR & RUPIAH & RINGGIT & BAHT & PESO \\
\hline YUAN & & & $\mathrm{V}$ & - & $\mathrm{V}$ & $\mathrm{V}$ & - & $\mathrm{V}$ & $\mathrm{V}$ \\
YEN & $\mathrm{V}$ & & $\mathrm{V}$ & - & $\mathrm{V}$ & $\mathrm{V}$ & - & $\mathrm{V}$ & $\mathrm{V}$ \\
WON & $\mathrm{V}$ & $\mathrm{V}$ & & $\mathrm{V}$ & $\mathrm{V}$ & $\mathrm{V}$ & - & $\mathrm{V}$ & $\mathrm{V}$ \\
EURO & - & $\mathrm{V}$ & $\mathrm{V}$ & & $\mathrm{V}$ & $\mathrm{V}$ & $\mathrm{V}$ & - & $\mathrm{V}$ \\
SING & - & $\mathrm{V}$ & $\mathrm{V}$ & - & & $\mathrm{V}$ & $\mathrm{V}$ & - & - \\
DOLLAR & & & & & & & $\mathrm{V}$ & $\mathrm{V}$ & $\mathrm{V}$ \\
RUPIAH & $\mathrm{V}$ & $\mathrm{V}$ & $\mathrm{V}$ & $\mathrm{V}$ & $\mathrm{V}$ & & $\mathrm{V}$ & $\mathrm{V}$ & - \\
RINGGIT & $\mathrm{V}$ & - & $\mathrm{V}$ & - & - & $\mathrm{V}$ & & $\mathrm{V}$ \\
BAHT & - & - & - & - & - & - & $\mathrm{V}$ & - & \\
PESO & - & $\mathrm{V}$ & $\mathrm{V}$ & $\mathrm{V}$ & $\mathrm{V}$ & $\mathrm{V}$ & - & - &
\end{tabular}

Source: IFS, World Bank (data processed).
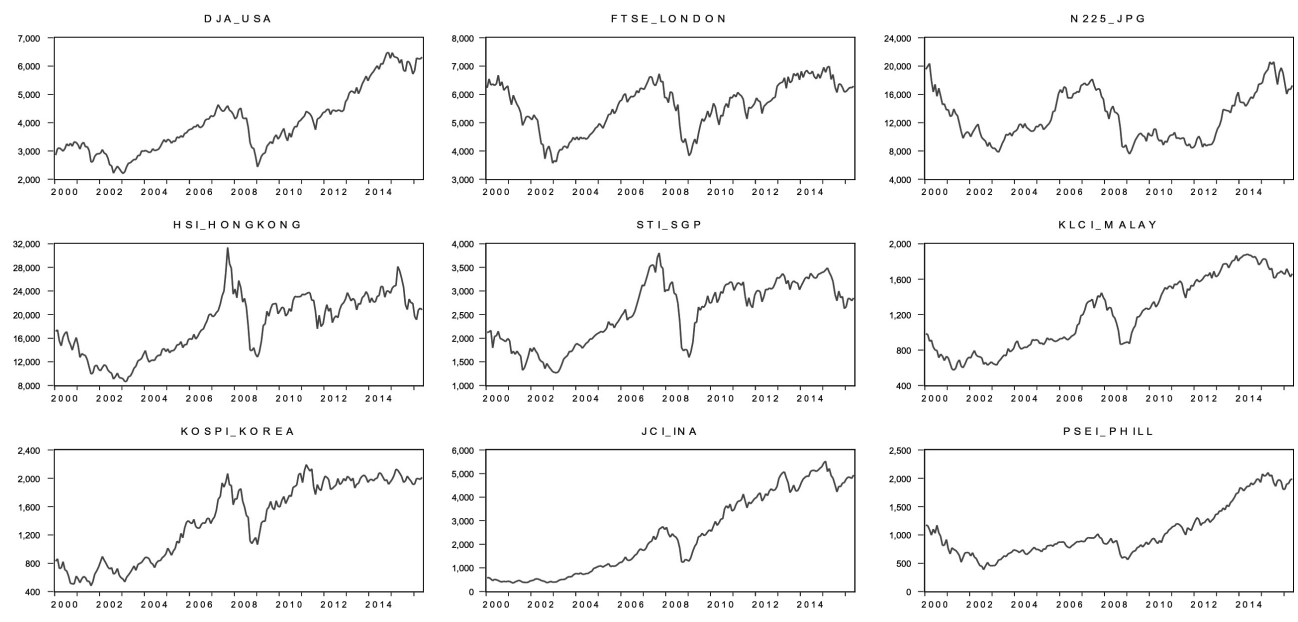

Figure 4. Movement of stock indices (2000M01-2016M05). Source: IFS, World Bank (data processed). 
Table 4. Engle-Granger cointegration test (2000M01-2007M12 and 2008M01-2016M05).

\begin{tabular}{lcccc}
\hline Dependent & $\tau$-Statistic & Probability* & Z-Statistic & Probability* \\
\hline Sample (adjusted): $2000 \mathrm{M} 02-2007 \mathrm{M} 2^{\mathrm{a}}$ & & & \\
VOLDJA & -11.04717 & 0.0000 & -107.1666 & 0.0000 \\
VOLFTSE & -11.77804 & 0.0000 & -112.2529 & 0.0000 \\
VOLN225 & -8.721248 & 0.0000 & -84.34860 & 0.0000 \\
VOLHSI & -8.118686 & 0.0000 & -79.19224 & 0.0000 \\
VOLSTI & -12.06771 & 0.0000 & -117.4155 & 0.0000 \\
VOLKLCI & -10.38735 & 0.0000 & -101.0770 & 0.0000 \\
VOLKOSPI & -8.258850 & 0.0000 & -80.96877 & 0.0000 \\
VOLJCI & -8.707544 & 0.0000 & -85.31091 & 0.0000 \\
VOLPSIE & -9.848600 & 0.0000 & -96.96465 & 0.0000 \\
Sample: $2008 M 01-2016 \mathrm{M} 05^{\mathrm{b}}$ & -8.980313 & 0.0000 & & \\
VOLDJA & -14.68942 & 0.0000 & -88.82707 & 0.0000 \\
VOLFTSE & -9.486608 & 0.0000 & -136.9682 & 0.0000 \\
VOLN225 & -14.13411 & 0.0000 & -132.76510 & 0.0000 \\
VOLHSI & -11.86782 & 0.0000 & -117.3961 & 0.0000 \\
VOLSTI & -11.49213 & 0.0000 & -113.4567 & 0.0000 \\
VOLKLCI & -11.11427 & 0.0000 & -110.4453 & 0.0000 \\
VOLKOSPI & -9.721341 & 0.0000 & -97.43779 & 0.0000 \\
VOLJCI & -9.264620 & 0.0000 & -93.04608 & 0.0000 \\
VOLPSIE & & & & 0.0000 \\
\hline VOLC & & & &
\end{tabular}

Notes: ${ }^{a}$ Observations included: 95 after adjustments. Null hypothesis: Series are not cointegrated. Cointegrating equation deterministics: C. Automatic lags specification based on Schwarz criterion

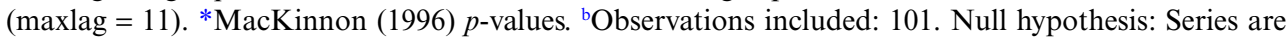
not cointegrated. Cointegrating equation deterministics: C. Automatic lags specification based on Schwarz criterion $(\operatorname{maxlag}=12) *$ MacKinnon (1996) $p$-values. VOL: Volatility, Source: finance.yahoo. com (data processed).

Table 5. Stock indices variance decomposition of four ASEAN countries (2000-16).

\begin{tabular}{|c|c|c|c|c|c|c|c|c|c|c|}
\hline Period & SE & $\begin{array}{l}\text { VOLD } \\
\text { JA }\end{array}$ & $\begin{array}{l}\text { VOLFT } \\
\text { SE }\end{array}$ & $\begin{array}{l}\text { VOLN } \\
225\end{array}$ & VOLHSI & VOLSTI & $\begin{array}{l}\text { VOLKL } \\
\text { CI }\end{array}$ & VOLJCI & $\begin{array}{l}\text { VOLKO } \\
\text { SPI }\end{array}$ & VOLPSIE \\
\hline \multicolumn{11}{|c|}{ Variance decomposition of VOLSTI } \\
\hline 1 & 114.9600 & 47.69286 & 11.43424 & 0.399156 & 2.222508 & 38.25124 & 0.000000 & 0.000000 & 0.000000 & 0.000000 \\
\hline 5 & 138.1525 & 34.60195 & 9.310296 & 2.406261 & 3.683320 & 28.93443 & 5.732481 & 9.580951 & 4.344760 & 1.405559 \\
\hline 10 & 160.5433 & 27.27902 & 10.52134 & 4.094994 & 3.695616 & 25.66420 & 9.201067 & 7.750331 & 9.359941 & 2.433493 \\
\hline \multicolumn{11}{|c|}{ Variance decomposition of VOLKLCI } \\
\hline 1 & 40.66464 & 428.25837 & 0.202599 & 0.283383 & 0.346999 & 6.537785 & 64.37087 & 0.000000 & 0.000000 & 0.000000 \\
\hline 5 & 48.23097 & 724.31232 & 2.171990 & 2.543803 & 1.832226 & 8.162921 & 48.98572 & 2.087606 & 7.394239 & 2.509173 \\
\hline 10 & 54.08151 & 120.68463 & 2.228770 & 2.873557 & 3.480435 & 7.418176 & 45 & 24 & 965 & 3.088402 \\
\hline \multicolumn{11}{|c|}{ Variance decomposition of VOLJCI } \\
\hline 1 & 139.4159 & 31.92667 & 0.750433 & 0.396513 & 10.9 & & & & 000 & 0.000000 \\
\hline 5 & 164.9735 & 28.56517 & 3.637239 & 4.997737 & 8.122945 & 10.12948 & 4.221935 & 34.22995 & 3.952738 & 2.142801 \\
\hline 10 & 189.9228 & 24.52058 & 4.619489 & 5.524466 & 9.937179 & 12.86817 & 6.201101 & 26.98155 & 4.049529 & 5.297934 \\
\hline \multicolumn{11}{|c|}{ Variance decomposition of VOLPSIE } \\
\hline 1 & 47.85577 & 775.47052 & 2.585713 & 2.855917 & 0.002269 & 0.992603 & 0.210547 & 0.031210 & 0.164168 & 17.68705 \\
\hline 5 & 56.39542 & 256.99567 & 3.507121 & 4.533257 & 1.312654 & 4.407288 & 9.884506 & 2.821910 & 2.180939 & 14.35666 \\
\hline 10 & 64.47664 & 46.38718 & 4.802660 & 9.713338 & 2.484115 & 6.124848 & 9.963242 & 2.880605 & 5.412743 & 12.23127 \\
\hline
\end{tabular}

Notes: SE, standard error. Cholesky ordering: VOLDJA VOLFTSE VOLN225 VOLHSI VOLSTI VOLKLCI VOLJCI VOLKOSPI VOLPSIE.VOL: Volatility.

Source: IFS, World Bank (data processed).

As shown in Table 5, in the period 2000-16, the stock indices of four ASEAN countries were predominantly influenced by the stock of the developed countries, especially the United States, with a contribution of between $20 \%$ and $75 \%$ with a forecast horizon of 10 peri- 
ods (monthly). Contributions from the regional stock indices to the movement of stock of ASEAN countrieswere still relatively small, with a maximum of about $12.86 \%$. Long-term relationship (cointegration) develops between the stock indices of developed countries and that offour ASEAN countries, in the periods before and after the 2008 financial crisis.

To determine whether there was a contagion effect, DCC testing in the period before and after the 2008 financial crisis was conducted, and the empirical results show that there was no significant increase in the DCC coefficient, indicating that contagion effect did not occur. What actually occurred was interdependence, as shown in Tables 6 and 7.

Table 6. DCC (2000-07).

\begin{tabular}{|c|c|c|c|c|c|c|c|c|c|c|}
\hline & $\begin{array}{l}\text { SDDJA_ } \\
\text { USA }\end{array}$ & $\begin{array}{l}\text { SDFTSE_ } \\
\text { LDN }\end{array}$ & $\begin{array}{l}\text { SDN225_ } \\
\text { JPG }\end{array}$ & $\begin{array}{l}\text { SDKLCI_ } \\
\text { MALAY }\end{array}$ & $\begin{array}{l}\text { SDHSI_ } \\
\text { HKG }\end{array}$ & $\begin{array}{l}\text { SDJCI_ } \\
\text { INA }\end{array}$ & $\begin{array}{l}\text { SDSTI_ } \\
\text { SGP }\end{array}$ & $\begin{array}{l}\text { SDPSEI_ } \\
\text { PHIL }\end{array}$ & $\begin{array}{l}\text { SDKOSPI } \\
\text { KOREA }\end{array}$ & \\
\hline $\begin{array}{l}\text { SDDJA_ } \\
\text { USA }\end{array}$ & 1.000 & 0.726 & 0.217 & 0.242 & 0.122 & 0.097 & 0.422 & 0.348 & 0.175 & \\
\hline $\begin{array}{l}\text { SDFTSE_ } \\
\text { LDN }\end{array}$ & 0.726 & 1.000 & 0.353 & 0.018 & 0.245 & -0.017 & 0.334 & 0.502 & 0.155 & \\
\hline $\begin{array}{l}\text { SDN225_ } \\
\text { JPG }\end{array}$ & 0.217 & 0.353 & 1.000 & 0.089 & 0.265 & -0.044 & 0.256 & 0.388 & 0.145 & \\
\hline $\begin{array}{l}\text { SDKLCI_ } \\
\text { MALAY }\end{array}$ & 0.242 & 0.018 & 0.089 & 1.000 & 0.370 & 0.396 & 0.652 & 0.111 & 0.205 & \\
\hline $\begin{array}{l}\text { SDHSI_ } \\
\text { HKG }\end{array}$ & 0.122 & 0.245 & 0.265 & 0.370 & 1.000 & 0.636 & 0.607 & 0.137 & 0.479 & \\
\hline $\begin{array}{l}\text { SDJCI_ } \\
\text { INA }\end{array}$ & 0.097 & -0.017 & -0.044 & 0.396 & 0.636 & 1.000 & 0.521 & -0.330 & 0.481 & \\
\hline $\begin{array}{l}\text { SDSTI_ } \\
\text { SGP }\end{array}$ & 0.422 & 0.334 & 0.256 & 0.652 & 0.607 & 0.521 & 1.000 & 0.147 & 0.461 & \\
\hline $\begin{array}{l}\text { SDPSEI_ } \\
\text { PHIL }\end{array}$ & 0.348 & 0.502 & 0.388 & 0.111 & 0.137 & -0.330 & 0.147 & 1.000 & 0.029 & \\
\hline $\begin{array}{l}\text { SDKOSPI_ } \\
\text { KOREA }\end{array}$ & 0.175 & 0.155 & 0.145 & 0.205 & 0.479 & 0.481 & 0.461 & 0.029 & 1.000 & \\
\hline Sum & 3.349 & 3.315 & 2.670 & 3.082 & 3.860 & 2.740 & 4.401 & 2.332 & 3.131 & \\
\hline Average & 0.2936 & 0.2894 & 0.2088 & 0.2603 & 0.3575 & 0.2175 & 0.4251 & 0.1665 & 0.2663 & 0.2761 \\
\hline
\end{tabular}

Source: IFS, World Bank (data processed). SD: Standard Deviation.

Table 7. DCC (2008-16).

\begin{tabular}{|c|c|c|c|c|c|c|c|c|c|c|}
\hline & $\begin{array}{l}\text { SDDJA_- } \\
\text { USA }\end{array}$ & $\begin{array}{l}\text { SDFTSE_ }_{\text {LDN }}\end{array}$ & $\begin{array}{l}\text { SDN225 } \\
\text { JPG }\end{array}$ & $\begin{array}{l}\text { SDKLCI } \\
\text { MALAY }\end{array}$ & $\begin{array}{l}\text { SDHSI_ } \\
\text { HKG }\end{array}$ & $\begin{array}{l}\text { SDJCI_ } \\
\text { INA }\end{array}$ & $\begin{array}{l}\text { SDSTI_ } \\
\text { SGP }\end{array}$ & $\begin{array}{l}\text { SDPSEI_ } \\
\text { PHIL }\end{array}$ & $\begin{array}{l}\text { SDKOSPI } \\
\text { KOREA }\end{array}$ & \\
\hline $\begin{array}{l}\text { SDDJA_ } \\
\text { USA }\end{array}$ & 1.000 & 0.469 & 0.424 & 0.445 & 0.324 & 0.297 & 0.425 & 0.542 & 0.280 & \\
\hline $\begin{array}{l}\text { SDFTSE_ } \\
\text { LDN }\end{array}$ & 0.469 & 1.000 & 0.033 & 0.704 & 0.534 & 0.523 & 0.678 & 0.257 & 0.621 & \\
\hline $\begin{array}{l}\text { SDN225 } \\
\text { JPG }\end{array}$ & 0.424 & 0.033 & 1.000 & 0.150 & 0.274 & 0.261 & 0.155 & 0.328 & -0.235 & \\
\hline $\begin{array}{l}\text { SDKLCI_ } \\
\text { MALAY }\end{array}$ & 0.445 & 0.704 & 0.150 & 1.000 & 0.619 & 0.542 & 0.768 & 0.253 & 0.636 & \\
\hline $\begin{array}{l}\text { SDHSI_ } \\
\text { HKG }\end{array}$ & 0.324 & 0.534 & 0.274 & 0.619 & 1.000 & 0.418 & 0.787 & 0.408 & 0.450 & \\
\hline $\begin{array}{l}\text { SDJCI_ } \\
\text { INA }\end{array}$ & 0.297 & 0.523 & 0.261 & 0.542 & 0.418 & 1.000 & 0.588 & 0.272 & 0.300 & \\
\hline $\begin{array}{l}\text { SDSTI_ } \\
\text { SGP }\end{array}$ & 0.425 & 0.678 & 0.155 & 0.768 & 0.787 & 0.588 & 1.000 & 0.330 & 0.617 & \\
\hline $\begin{array}{l}\text { SDPSEI_ } \\
\text { PHIL }\end{array}$ & 0.542 & 0.257 & 0.328 & 0.253 & 0.408 & 0.272 & 0.330 & 1.000 & 0.229 & \\
\hline $\begin{array}{l}\text { SDKOSPI_ } \\
\text { KOREA }\end{array}$ & 0.280 & 0.621 & -0.235 & 0.636 & 0.450 & 0.300 & 0.617 & 0.229 & 1.000 & \\
\hline Sum & 4.206 & 4.819 & 2.389 & 5.118 & 4.815 & 4.203 & 5.349 & 3.620 & 3.898 & \\
\hline Average & 0.401 & 0.477 & 0.174 & 0.515 & 0.477 & 0.400 & 0.544 & 0.328 & 0.362 & 0.3893 \\
\hline
\end{tabular}

Source: IFS, World Bank (data processed). 
Based on the DCC after the 2008 financial crisis, there was an increase of interdependence between the stock indices of the four ASEAN countries and the developed countries, except United Kingdom. It shows that after the 2008 financial crisis, the openness of capital flows across countries increased, resulting in the greater spread of global risk through portfolio diversification across countries. On the one hand, this increase provides convenience for global funding; on the other hand, the economic vulnerability also increases.

\subsection{Policy interest rates}

Based on Figure 5, the Central Bank policy interest rates in the five ASEAN countries and the United States tend to decrease, especially after the 1997 financial crisis. According to the Engle-Granger cointegration test policy rates in Table 8, a long-term equilibrium relationship did not occur in all the five ASEAN countries, but only cointegration occurred in Singapore and Malaysia.

As seen in Table 9, there was an increase in the DCC coefficient in the three periods of the analysis. This indicates the presence of a strong relationship between the monetary policies in each country and those in other countries. It also indicates the increasing coordination of monetary policies among the five ASEAN countries. The correlation coefficient of the US policy rates increased sharply, especially after the 2008 financial crisis. These indicate that the US monetary policy was still used as a reference by the five ASEAN countries.

USA

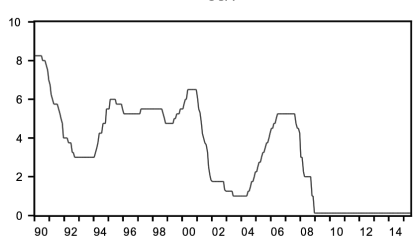

INDONESIA

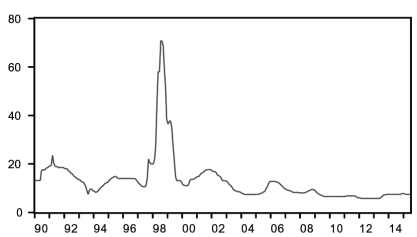

SINGAPORE

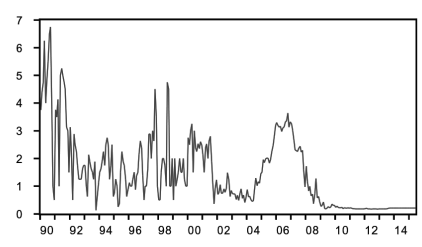

THAILAND

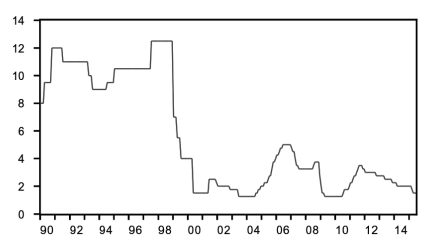

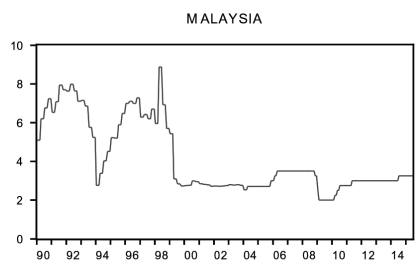

PHILIPPINES

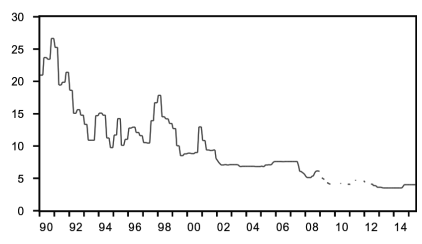

Figure 5. Movement policy interest rates. Source: IFS, World Bank (data processed).

Table 8. Engel-Granger cointegration test policy rates in five ASEAN countries and the United States.

\begin{tabular}{lllll}
\hline Dependent & $\tau$-Statistic & Probability* & Z-Statistic & Probability* \\
\hline United States & -3.215680 & 0.6367 & -22.01180 & 0.5429 \\
Singapore & -8.755051 & 0.0000 & -119.7866 & 0.0000 \\
Malaysia & -4.499010 & 0.0946 & -37.95018 & 0.0833 \\
Indonesia & -3.995860 & 0.2492 & -34.02247 & 0.1440 \\
Thailand & -4.195686 & 0.1753 & -32.93174 & 0.1678 \\
Philippines & -4.016446 & 0.2404 & -28.86497 & 0.2766 \\
\hline
\end{tabular}

Notes: Series: United States, Singapore, Malaysia, Indonesia, Thailand, Philippines.

Sample: 1990M01-2015M06. Included observations: 279. Null hypothesis: Series are not cointegrated. Cointegrating equation deterministics: C. Automatic lags specification based on Schwarz criterion (maxlag = 15). *MacKinnon (1996) $p$-values.

Source: IFS, World Bank (data processed). 
Table 9. DCC of policy interest rates (1990-2015) on average from each country.

\begin{tabular}{|c|c|c|c|c|c|c|c|c|c|c|}
\hline & $\begin{array}{l}\mathrm{DCC}_{-} \\
\text {CHINA }\end{array}$ & $\begin{array}{l}\mathrm{DCC}_{-} \\
\mathrm{IND}\end{array}$ & $\begin{array}{l}\mathrm{DCC}_{-} \\
\mathrm{JPG}\end{array}$ & $\begin{array}{l}\mathrm{DCC}_{-} \\
\mathrm{KOR}\end{array}$ & $\begin{array}{l}\mathrm{DCC}_{-} \\
\text {MALAY }\end{array}$ & $\begin{array}{l}\mathrm{DCC}_{-} \\
\text {PHIL }\end{array}$ & $\begin{array}{l}\mathrm{DCC}_{-} \\
\mathrm{SGP}\end{array}$ & $\begin{array}{l}\mathrm{DCC}_{-} \\
\text {THAI }\end{array}$ & $\begin{array}{l}\mathrm{DCC}_{-} \\
\mathrm{UK}\end{array}$ & $\begin{array}{l}\mathrm{DCC}_{-} \\
\mathrm{USA}\end{array}$ \\
\hline $\begin{array}{l}\text { 1990M01 } \\
\text { 1997M07 }\end{array}$ & 0.0322 & 0.0897 & 0.1278 & 0.3410 & 0.0239 & 0.3488 & 0.3420 & 0.1867 & 0.2686 & 0.0100 \\
\hline $\begin{array}{l}\text { 1998M01 } \\
2007 \mathrm{M} 12\end{array}$ & 0.4289 & 0.2845 & 0.3753 & 0.3836 & 0.4956 & 0.3758 & 0.4470 & 0.3885 & 0.3262 & 0.0998 \\
\hline $\begin{array}{l}2008 \mathrm{M} 01 \\
2015 \mathrm{M} 05\end{array}$ & 0.7104 & 0.5539 & 0.7877 & 0.8442 & 0.7682 & 0.7691 & 0.8477 & 0.6764 & 0.8222 & 0.8084 \\
\hline
\end{tabular}

Source: IFS, World Bank (data processed).
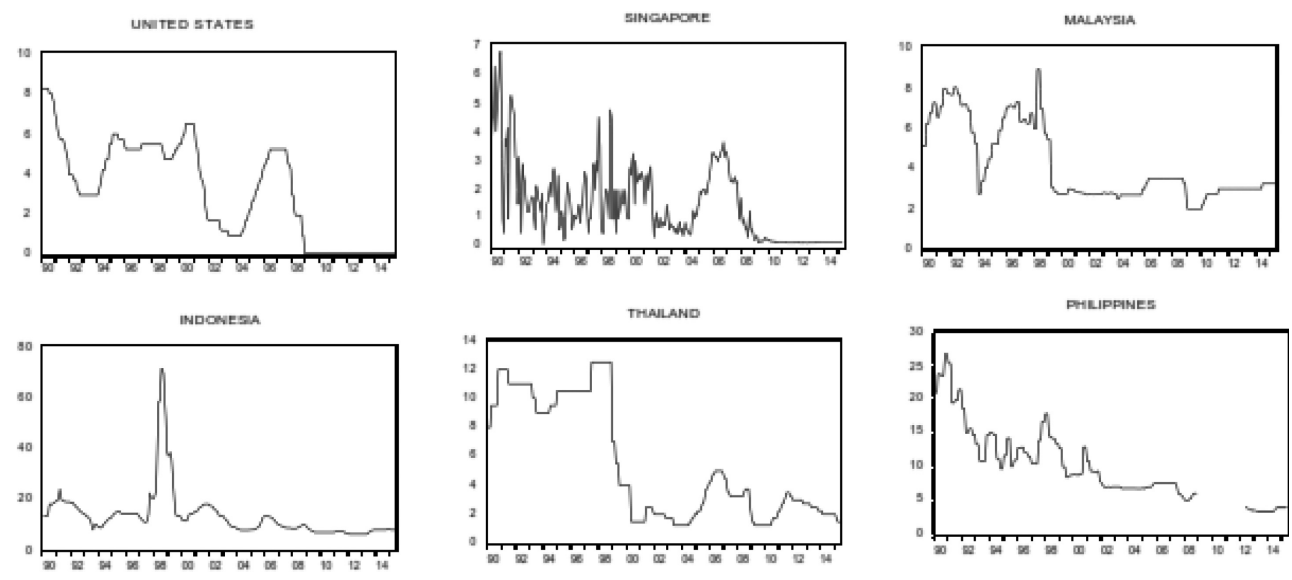

Figure 6. Money market rates in the five ASEAN countries and the United States. Source: IFS, World Bank (data processed).

Table 10. Engel-Granger cointegration test of money market rates.

\begin{tabular}{lllll}
\hline Dependent & $\tau$-Statistic & Probability* & Z-Statistic & Probability* \\
\hline United States & -4.052550 & 0.7464 & -24.27781 & 0.8566 \\
European Union & -3.994672 & 0.7695 & -26.37325 & 0.7898 \\
Japan & -4.122106 & 0.7174 & -27.51796 & 0.7483 \\
China & -5.543007 & 0.1374 & -44.72646 & 0.1292 \\
Korea & -2.134344 & 0.9993 & -11.78542 & 0.9983 \\
Singapore & -5.097402 & 0.2750 & -38.26990 & 0.3087 \\
Malaysia & -3.476455 & 0.9224 & -176.8311 & 0.0000 \\
Indonesia & -3.022637 & 0.9790 & -60.78869 & 0.0035 \\
Philippines & -5.434343 & 0.1651 & -42.44309 & 0.1810 \\
Thailand & -5.712595 & 0.1014 & -46.43230 & 0.0984 \\
\hline
\end{tabular}

Notes: Null hypothesis: Series are not cointegrated. Cointegrating equation deterministics: C. Automatic lags specification based on Schwarz criterion (maxlag $=11)$. ${ }^{*}$ MacKinnon (1996) $p$-values.

Source: IFS, World Bank (data processed).

\subsection{Money market}

Figure 6 shows that the movement of money market interest rates does not have the same pattern. This is reinforced by the cointegration test results, which in the long run shows there is no relationship of money market interest rates in the five ASEAN countries and those in the five developed countries (see Table 10). The absence of cointegration relationships shows 
Table 11. Correlation coefficient of money market rates in three periods.

\begin{tabular}{ll}
\hline Period & Correlation coefficientin average \\
\hline 1994Q1-1997Q3 & 0.35 \\
1998Q1-2007Q4 & 0.41 \\
2008Q1-2014 Q2 & 0.56 \\
\hline
\end{tabular}

Source: IFS, World Bank (data processed).

Table 12. Variance decomposition of money market rates (1994Q1-2014Q2).

\begin{tabular}{|c|c|c|c|c|c|c|c|}
\hline Period & SE & D(USA) D(EU) & $\begin{array}{ll}\mathrm{D} & \mathrm{D} \\
(\mathrm{JAPAN}) & (\mathrm{CHINA}\end{array}$ & $\begin{array}{l}\text { D } \\
\text { (KOREA }\end{array}$ & $\begin{array}{l}\text { D } \\
(\text { SING })\end{array}$ & $\begin{array}{l}\text { D } \\
\text { (MALAY) }\end{array}$ & D(INA) D(PHIL) D(THAI) \\
\hline \multicolumn{8}{|c|}{ Variance decomposition of $\mathrm{D}$ (SING) } \\
\hline 1 & 0.248879 & 21.182388 .78336 & 811.026467 .128446 & 3.709676 & 48.16967 & 70.000000 & 0.0000000 .0000000 .000000 \\
\hline 5 & 0.479858 & 20.8159710 .4780 & 829.149586 .182966 & 2.814854 & 18.69895 & 52.459190 & 2.2085201 .3634225 .828464 \\
\hline 10 & 0.615699 & 23.8588612 .0839 & 520.4447310 .29808 & 2.603831 & 18.38128 & 82.074972 & 3.1850101 .9530925 .116194 \\
\hline \multicolumn{8}{|c|}{ Variance decomposition of D(MALAY) } \\
\hline 1 & 0.151211 & 0.42337813 .5049 & 614.492592 .196494 & 40.20865 & 5.827138 & 823.34679 & 0.0000000 .0000000 .000000 \\
\hline 5 & 0.416577 & 79.1985885 .90774 & 916.4672010 .28322 & 11.78560 & 29.76712 & 211.52986 & 1.8473720 .4355742 .777717 \\
\hline 10 & 0.663802 & 26.968174 .46478 & 49.7840508 .775853 & 8.542639 & 25.92369 & 95.921986 & 5.2880411 .5215552 .809228 \\
\hline \multicolumn{8}{|c|}{ Variance decomposition of D(INA) } \\
\hline 1 & 2.054912 & 29.302700 .50016 & $98.72 \mathrm{E}-051.241160$ & 1.354811 & 54.48772 & 20.837615 & 12.275740 .0000000 .000000 \\
\hline 5 & 3.966181 & 21.679783 .10129 & $34.983198 \quad 10.42426$ & 11.82745 & 25.60559 & 94.625691 & 7.8479911 .8567538 .047991 \\
\hline 10 & 5.780639 & 24.473872 .83844 & 47.33512510 .26036 & 7.872738 & 27.6573 & 44.336255 & 7.7425581 .7330365 .750272 \\
\hline \multicolumn{8}{|c|}{ Variance decomposition of $\mathrm{D}$ (PHIL) } \\
\hline 1 & 0.705387 & 72.3413950 .00800 & 730.270466 .625379 & 4.807545 & 38.29211 & 17.076287 & 3.8809186 .6979030 .000000 \\
\hline 5 & 1.352914 & 45.0778092 .19616 & 129.006519 .307887 & 7.453509 & 33.26384 & 45.567594 & 2.6028604 .0885401 .435291 \\
\hline 10 & 1.603841 & 7.3008633 .33173 & 826.3703210 .49192 & 9.103535 & 28.35473 & 34.725600 & 3.1909503 .9309913 .199350 \\
\hline \multicolumn{8}{|c|}{ Variance decomposition of $\mathrm{D}$ (THAI) } \\
\hline 1 & 0.508058 & 5.84455924 .7204 & 20.2334921 .379816 & 5.036296 & 25.17341 & 11.329327 & 2.4921532 .92194730 .86858 \\
\hline 5 & 1.579542 & 210.634365 .70009 & $817.65382 \quad 15.10879$ & 7.911774 & 25.31946 & 67.401335 & 2.2431972 .5037455 .523430 \\
\hline 10 & 1.906556 & 616.398485 .41081 & $213.87307 \quad 14.85211$ & 7.812366 & 24.97486 & 66.046350 & 3.6093553 .1295703 .893024 \\
\hline
\end{tabular}

that in the long term movement of money market rates does not converge to a certain value, but it is more likely to be determined by the supply and demand for money in each country.

Based on the correlation coefficients in the three different periods as shown in Table 11, there was no increase in the correlation coefficient on a large scale after the 2008 financial crisis. Thus, contagion effect did not occur and only interdependence was noted.

Regionally, money market rates in Singapore had the most impact on movements in the five ASEAN countries; globally, the US money market rates were still dominant, as shown in Table 12.

\section{CONCLUSIONS}

1. Exchange rate fluctuations had the same direction in the five ASEAN and five developed countries, indicating a strong global relationship among currencies. However, empirical evidence indicated the occurrence of interdependence but not contagion.

2. Stock indices in the four ASEAN countries were predominantly influenced by the stock indices from the developed countries, especially the United States. Contributions from regional indices to the movement of stocks of ASEAN countries were relatively small. There was a long-term relationship (cointegration) between the stock of the developed countries and that of the ASEAN countries, in the periods before and after the 2008 financial crisis. The DCC coefficient showed no contagion effect and only interdependence among the stock markets. 
3. Correlation of policy interest rates between the five countries of ASEAN and the United States increased after the crisis in 1997 and still continued after the 2008 financial crisis. The increase indicates that the US monetary policy was still used as a reference by the five ASEAN countries.

4. The movement of money market interest rates did not have the same pattern for the five ASEAN and the five developed countries. It shows that in the long term, the movement is more likely to be determined by the supply and demand of money in each country.

\section{REFERENCES}

Ahlgren, N. \& Antell, J. (2010). Stock market linkages and financial contagion: A cobreaking analysis. Quarterly Review of Economics and Finance, 50(2), 157-166. doi:10.1016/j.qref.2009.12.004.

Claessens, S., \& Forbes, K., (2004). International Financial Contagion: The Theory, Evidence and Policy Implications. The IMF's Conference: Role in Emerging Market Economies: Reassessing the Adequacy of its Resources' organized by RBWC, DNB and WEF.

Dornbusch, R., Park Y.C. \& Claessens, S. (2000). Contagion: Understanding how it spreads. The World Bank Research Observer, 15(2), 177-197. doi:10.1093/wbro/15.2.177.

Dungey, M. \& Tambakis, D. (2003). Financial contagion: What do we mean? What do we know? Available at: http://hpi.uw.hu/pdf/Dungey-Tambakis\%20Presentation\%20Dubai\%2003.pdf (accessed May 2017).

Forbes, K. \& Rigobon,R.(2001). Contagion in Latin America: Definition, measurement, and policy implications. Economia Journal of The Latin America and Caribbean Economic Association, 1(2),1-46.

Forbes, K. \& Rigobon, R. (2002). No contagion, only interdependence: Measuring stock market comovements. Journal of Finance, 57(5), 2223-2261.

Gómez, M., \& Rivero, S. (2014). Causality and contagion in EMU sovereign debt markets. International Review of Economics and Finance, 33(2014), 12-27.

Gray, A.(2015).Understanding interconnectedness risk, to build amore resilient financial system. A white paper to the industry.

Kaminsky, et.all., (2003). The unholy trinity of financialcontagion. Journal of Economic PerspectivesVolume 17, Number 4-Fall 2003-Pages 51-74.

Kaminsky, G.L., \& Reinhart, C.M. (2000). On crises, contagion, and confusion. Journal of International Economics, 51, 145-168. doi:10.1016/S0022-1996(99)00040-9.

Kenourgios, D. (2014). On financial contagion and implied market volatility. International Review of Financial Analysis, 34(C), 21-30.

Luchtenberg, K.F. \& Vu, Q.V. (2015). The 2008 financial crisis: Stock market contagion and its determinants.Journal of Research in International Business and Finance, 33(2015), 178-203.

Morales, L. \& O'Callaghan, B.,A.,. (2014). The global financial crisis: World market or regional contagion effects? International Review of Economics and Finance, 29, 108-131. doi: http://dx.doi. org/10.1016/j.iref.2013.05.010.

Podlich, N. \& Wedow, M. (2014). Crossborder financial contagion to Germany: How important are OTC dealers? International Review of Financial Analysis, 33, 1-9. doi:10.1016/j.irfa.2013.07.008.

Shen, P.L., Li, W., Wang, X.-T., and Su, C.-W. (2015). Contagion effect of the European financial crisis on China's stock market: Interdependence and pure contagion. Economic Modelling, 50(2015), 193-199.

Unsal, D.F. \& Jain-Chandra, S. (2012). The effectiveness of monetary policy transmission under capital inflows: Evidence from Asia. IMF Working Paper WP/12/265. 